ACCEPTED MANUSCRIPT

\title{
Tissue engineering of retina through high resolution 3-dimentional inkjet bioprinting
}

To cite this article before publication: Elahe Masaeli et al 2019 Biofabrication in press https://doi.org/10.1088/1758-5090/ab4a20

\section{Manuscript version: Accepted Manuscript}

Accepted Manuscript is "the version of the article accepted for publication including all changes made as a result of the peer review process, and which may also include the addition to the article by IOP Publishing of a header, an article ID, a cover sheet and/or an 'Accepted Manuscript' watermark, but excluding any other editing, typesetting or other changes made by IOP Publishing and/or its licensors"

This Accepted Manuscript is @ 2019 IOP Publishing Ltd.

During the embargo period (the 12 month period from the publication of the Version of Record of this article), the Accepted Manuscript is fully protected by copyright and cannot be reused or reposted elsewhere.

As the Version of Record of this article is going to be / has been published on a subscription basis, this Accepted Manuscript is available for reuse under a CC BY-NC-ND 3.0 licence after the 12 month embargo period.

After the embargo period, everyone is permitted to use copy and redistribute this article for non-commercial purposes only, provided that they adhere to all the terms of the licence https://creativecommons.org/licences/by-nc-nd/3.0

Although reasonable endeavours have been taken to obtain all necessary permissions from third parties to include their copyrighted content within this article, their full citation and copyright line may not be present in this Accepted Manuscript version. Before using any content from this article, please refer to the Version of Record on IOPscience once published for full citation and copyright details, as permissions will likely be required. All third party content is fully copyright protected, unless specifically stated otherwise in the figure caption in the Version of Record.

View the article online for updates and enhancements. 


\section{Tissue Engineering of Retina through High Resolution 3-}

\section{dimentional Inkjet Bioprinting}

4 Elahe Masaeli ${ }^{[1,2]}$, Valérie Forster ${ }^{[3]}$, Serge Picaud ${ }^{[3]}$, Fereshte Karamali ${ }^{[1]}$, Mohammad

5 Hossein Nasr-Esfahani ${ }^{[1]}$, Christophe Marquette ${ }^{[2]}$

${ }^{[1]}$ Department of Cellular Biotechnology, Cell Science Research Center, Royan Institute for

8 Biotechnology, ACECR, Isfahan, Iran.

9

10

[2] 3d.FAB, Univ Lyon, Université Lyon1, CNRS, INSA, CPE-Lyon, ICBMS, UMR 5246, Bat.

11 Lederer, 1 rue Victor Grignard, 69100 Villeurbanne, France.

$13 \quad{ }^{[3]}$ Institut de la Vision, 17 rue Moreau, 75012 Paris.

KEYWORDS: Retina Pigmented Epithelium; Photoreceptors; Bioprinting; Tissue Engineering 
1 ABSTRACT: The mammalian retina contains multiple cellular layers, each carrying out a specific

2 task. Such a controlled organization should (Yue et al., 2015)be considered as a crucial factor for

3 designing retinal therapies. The maintenance of retinal layered complexity through the use of

4 scaffold-free techniques has recently emerged as a promising approach for clinical ocular tissue

5 engineering.

6 In an attempt to fabricate such layered retinal model, we are proposing herein a unique inkjet

7 bioprinting system applied to the deposition of a photoreceptor cell (PRs) layer on top of a

8 bioprinted retinal pigment epithelium (RPE), in a precise arrangement and without any carrier

9 material. The results showed that, after bioprinting, both RPE and PRs were well positioned in a

10 layered structure and expressed their structural markers, which was further demonstrated by ZO1,

11 MITF, rhodopsin, opsin B, opsin R/G and PNA immunostaining, 3 days after bioprinting. We also

12 showed that considerable amounts of human vascular endothelial growth factor (hVEGF) were

13 released from the RPE printed layer, which confirmed formation of a functional RPE monolayer

14 after bioprinting. Microstructures of bioprinted cells as well as phagocytosis of photoreceptor outer

15 segments by apical RPE microvilli was finally established through transmission electron

16 microscopy (TEM) imaging. In summary, using this carrier-free bioprinting method, it was

17 possible to develop a reasonable in vitro retina model for studding some sight-threatening diseases

18 such as age-related macular degeneration (AMD) and retinitis pigmentosa (RP).

46
47
48
49
50
51
52
53
54
55
56
57
58
59


2 The retina is a complex photosensitive layer of the central nervous system, which covers inner

3 surface of eye cup and is vital for maintenance of vision (Singh et al., 2018). Vertebrate retina

4 contains different parallel cellular layers, including inner neuroepithelial and outer pigmented

5 epithelial layers. Among neural cells, light-sensing photoreceptors, i.e. cones and rods, are

6 responsible of visual phototransduction in eye. To begin the process of seeing, light enters the eye,

7 traverse most of inner layers, and finally impinges on the outer segments of photoreceptors.

8 Photoreceptors then transduce photon energy into electric signals that are conveyed to other retinal

9 neurons. The retinal pigment epithelium (RPE) consists of a cell monolayer situated between the

10 photoreceptors and the choroid. This epithelium supports and insulates the photoreceptors to

11 increase visual resolution at high light levels.

12 Since these photoreceptors are fully differentiated neurons lacking regenerative capacity (Tropepe

13 et al., 2000), dysfunction of these retinal layers leads to several irreversible sight-threatening

14 diseases such as age-related macular degeneration (AMD) and retinitis pigmentosa (RP). These

15 diseases are then particularly attractive/challenges for regenerative medicine developments

16 (Ramsden et al., 2013; Trese et al., 2012).

17 In these diseases, areas of RPE atrophy are associated with photoreceptor degeneration. Hence,

18 most of stem cell-based therapies focus on replacing RPE to maintain normal function of

19 photoreceptors or attempt to restore their integrity in the affected eye (Trese et al., 2012).

20 Unfortunately, several challenges such as selection of cell delivery method, limited integration of

21 grafted cells and incomplete differentiation to a specific fate hinder the success of function

22 regeneration (Yao et al., 2011). 
1 Accordingly, in recent studies, tissue engineering based strategies have been widely applied as an

2 alternative approach to address aforementioned problems. Synthetic and natural polymeric

3 scaffolds with well-defined requirements, such as biocompatibility in the sub-retinal space, ultra-

4 fine structure, and appropriate physico-mechanical properties for implantation, has been

5 introduced to guide retinal repair (Yao et al., 2011; Trese et al., 2012; Tomita et al., 2005).

6 Nevertheless, inflammatory side effects and loose integration of RPE layer to the underlying

7 choroid, that sustains the RPE and photoreceptors' function, are two main challenges in applying

8 biodegradable scaffolds for retinal regeneration.

9 Cell sheet engineering is another alternative, which does not rely on a polymer scaffold. Such a

10 strategy is based on harvesting cultured cells as intact sheets along with their extracellular matrix

11 (ECM), and combine them to form tissue-like structures (Mokhtarinia et al., 2018). In previous

12 studies, cellular monolayers from different cell sources have been generated using conventional

13 scaffold-free engineering approaches to treat retinal degenerative diseases (Yaji et al., 2009; Yang

14 et al., 2006; Umemoto et al., 2013). However, retinal cellular complexity, as a crucial requirement

15 for effective function of engineered grafts, has not been addressed in these studies.

16 Additive manufacturing for direct patterning and depositing of living materials, known as 3D

17 bioprinting, has been increasingly used to fabricate cell based 3D structures (De Maria et al., 18 2017). Indeed, the capability of 3D bioprinter systems (laser-based, inkjet-based or extrusion-

19 based) for layer-by-layer precise positioning of bio-components in cell-friendly conditions bring

20 various advantages over exiting fabrication technologies, the principal one being the capability to 21 generate complex and predefined 3D geometries (Tasoglu and Demirci, 2013). The structures

22 obtained through 3D bioprinting were then shown to be useful for the development of 3D 23 constructs with tailored biological heterogeneity, directly apply in tissue engineering and 
1 regenerative medicine studies (Kolesky et al., 2014; Xu et al., 2013; Pourchet et al., 2017), but

2 also cell-based sensors (Dias et al., 2014), drug screening (Jorge et al., 2012) and tumor models

3 production(Yu et al., 2014). The definition of these geometries is directly related to the bioprinting

4 technique used, for example, the highest precision (single cell deposition) reached using laser-

5 based systems (Guillotin and Guillemot, 2011; da Silva et al.), high resolution (tenth of cells or $\mathrm{nL}$

6 drop) using inkjet-based technique and medium resolution but large volume geometries using

7 extrusion-based bioprinters. The herein reported study aimed at demonstrating the capability of

8 inkjet-based bioprinting to recapitulate retinal components. Indeed, layer-by-layer bioprinting

9 process matches nicely with the native multilayered anatomy of the retina and cells precise

10 deposition through inkjet bioprinting might help control their subsequent interactions.

11 To the best of our knowledge, there have been very few reports on attempt to construct

12 multilayered tissue-engineered retinal tissue through bioprinting. Two studies in the field of

13 successive bioprinting of retinoblastoma cells on RPE layer are among the seldom efforts made in

14 this area (Shi et al., 2017; Shi et al., 2018).

15 The objective of the present study was then to develop a three-dimensional retina model, which

16 may have clinical implications for retinal regeneration therapies. In order to fabricate this

17 construct, inkjet-based bioprinting strategy was applied to deposit matured and differentiated

18 photoreceptors on RPE layer in a predefined arrangement to create complex double-cell sheets. A

19 thin layer of gelatin methacrylate (GelMa) was also explored to mimic Bruch's membrane before

20 RPE deposition.

21

22

23 


\section{MATERIALS \& METHODS}

Gelatin Methacrylate (GelMa) Solution Preparation and Coating

3 To simulate the basement membrane of RPE (i.e. Bruch's membrane), GelMa coating was applied

4 to circular glass coverslips (0.5 mm thick, $17 \mathrm{~mm}$ diameter, T\&Q, China), subsequently used as

5 substrate for bioprinting. Coating solution was prepared by dissolving $2 \mathrm{mg}$ of GelMa (Sigma

6900496 , France) in $10 \mathrm{~mL}$ of a $1.5 \%$ (w/v) solution of Irgacure 2959 (Sigma 410896, France) in

7 dimethyl sulfoxide (Merck 102952, Germany) for 30 minutes at $55{ }^{\circ} \mathrm{C}$. Once dissolved, the

8 solution was centrifuged to remove air bubbles. Stock GelMa solution was stored in a light tight

9 container at $2-8{ }^{\circ} \mathrm{C}$ to prevent unintentional crosslinking by ambient light. Prior GelMa thin layer

10 spin coating, the circular glass coverslips were degreased with acetone, rinsed with deionized water

11 and air dried. $200 \mu \mathrm{l} \mathrm{GelMa}$ stock solution was then dropped onto each cover glass coverslips

12 rotating at $3000 \mathrm{rpm}$ and coated for 30 seconds using a WS-650MZ spin coater (Laurell, USA).

13 After spin coating, GelMa polymerization was performed by placing the coated coverslips in a UV

14 chamber (BLX-E254, Bio-Link, Fisher Biotec, Australia ) for 30 minutes.

RPE Cell Culture and Labeling

17 RPE cell line ARPE-19 (ATCC CRL-2302, France) was cultured in DMEM-F12 medium (ATCC

18 30-2006, France) supplemented with 10\% fetal bovine serum (FBS, Gibco 10270, France) and 1\%

19 penicillin/streptomycin (Gibco 15140, France). Before inkjet bioprinting, RPE cells were stained

20 with fluorescent dye (PKH67 GL, Sigma, France) according to the manufacturer's instructions.

21 Briefly, $1 \times 10^{7}$ cells were suspended in $1 \mathrm{ml} 2 \times 10^{-6} \mathrm{M}$ PKH 67 solution in diluent $\mathrm{C}$ (provided in

22 the staining kit), and incubated for 1-5 minutes at room temperature with periodic mixing. The

23 excess staining reagents were first diluted with an equal volume of culture medium supplemented 
with $10 \%$ FBS, then discarded through centrifugation for 10 minutes at $800 \mathrm{rpm}$. The cellular pellet

2 was recovered and washed again with supplemented culture medium.

\section{Isolation of Photoreceptors (PRs)}

4 Adult pig ocular globes were obtained from the surgical school of University Lyon 1 immediately

5 after euthanasia and transported to the laboratory in ice-cold $\mathrm{CO}_{2}$-independent Dulbecco's

6 modified Eagle's medium (DMEM) (Gibco 18045, France) supplemented with $10 \mathrm{mg} / \mathrm{ml}$

7 gentamicin (Gibco 15710, France). Dissection of the retina from ocular globes was performed in

8 our laboratory, as described previously (Luo et al., 2001).

9 Briefly, after immersion of the whole globe in two successive disinfection baths (PhagoSpray,

10 Phagogene, France) and one $\mathrm{CO}_{2}$-independent medium, the cornea with lens and vitreous attached

11 was removed. Retina layer was separated from the posterior eye cup, cut into small pieces (1-2

$12 \mathrm{~mm}$ ) and subsequently washed twice with warm glucose solution in phosphate buffer saline (PBS,

13 Gibco 14190, France) (1 g/L). Afterward, retina pieces were incubated in 4 Unit activated papain

14 solution (Worthington LSO 3124, USA) at $37^{\circ} \mathrm{C}$ for 20 minutes and shaken gently every 10

15 minutes. To stop digestion step, $1 \mathrm{ml}$ of Neurobasal-A medium (Nb-A, Invitrogen 10888, France)

16 supplemented with 10\% FBS and DNase I was applied. The tissue fragments were dissociated by

17 gentle shaking of the tube for 10 seconds, after which the suspension was allowed to settle for 2

18 minutes. The supernatant containing PRs was carefully harvested, fresh $\mathrm{Nb}-\mathrm{A}$ was added and the

19 gentle shaking repeated. The suspension was allowed to settle for another 2 minutes, after which

20 the supernatant was collected and pooled with the first one. The pellet was discarded, and the

21 pooled supernatants were centrifuged for 5 minutes at $800 \mathrm{rpm}$ and finally re-suspended in $\mathrm{Nb}-\mathrm{A}$

22 supplemented with 2\% B27 (Invitrogen 17504, France). Viable cell number was estimated after

23 trypan blue vital dye exclusion. Schematic of PRs isolation has been illustrated in Figure 2-A. 
2 Micro-patterning of RPE and PRs arrays was performed using a piezoelectric inkjet dispenser S3

3 sciFLEXARRAYER (Scienion AG, Germany) used here as a non-contact bioprinter. The

4 instrument has a three axis micro-positioning system (accuracy $10 \mu \mathrm{m}$ ) and is equipped with an 80

$5 \mu \mathrm{m}$ diameter glass nozzle. The vertical separation between the nozzle and the substrate was

6 typically $500 \mu \mathrm{m}$. A stroboscopic camera allows visual monitoring to adjust piezo voltages and

7 pulse durations for reliable droplet ejection. Single drop ejected from the nozzle has a mean volume

8 of $300 \mathrm{pL}$ under our experimental conditions. For RPE bioprinting, arrays of 50 deposition

9 locations of 52 drops were dispensed within an array of $10 \times 10$ spots (spot pitch of $800 \mu \mathrm{m}$ ). For

10 PRs bioprinting, arrays of 100 deposition locations of 52 drops were dispensed within an array of

$1110 \times 10$ spots (spot pitch of $800 \mu \mathrm{m})($ Supplementary information 1). Bioprinted constructs were

12 cultivated in the culture medium of PRs containing $\mathrm{Nb}-\mathrm{A}$ supplemented with $2 \% \mathrm{~B} 27$.

\section{Cellular viability assay}

15 Cell population growth assessments were performed using an in vitro viability assay kit (Sigma

16 TOX8, France) based on resazurin enzymatic reduction by cell metabolic activity. Briefly, for each

17 proliferation assay, cell culture supernatant was harvested and $10 \%(\mathrm{v} / \mathrm{v})$ of resazurin dye was

18 added to the culture medium. According to manufacturer's protocol, RPE cells with high density

19 and enough metabolic activity were incubated just for 2 hours at $37^{\circ} \mathrm{C}$, and subsequently assessed

20 spectrophotometrically by monitoring the decrease in absorbance at a wavelength of $600 \mathrm{~nm}$.

21 Measures of the absorbance of 96-well plates were also performed at a reference wavelength of

$22690 \mathrm{~nm}$ and subtracted from the $600 \mathrm{~nm}$ measurements. Results of these cell population growth

23 assays were then given as "Neat Absorbance" corresponding to OD600-OD 690 . 


\section{Actin Cytoskeleton Staining}

2 Specific staining of cellular actin cytoskeleton was performed on bioprinted samples using 2 steps

3 protocol. First, cellular constructs were fixed with 3.7\% (v/v) paraformaldehyde (Sigma P6148,

4 France) diluted in PBS for 30 minutes at room temperature and then washed 3 times with PBS.

5 Afterward, the fixed constructs were permeabilized by Triton X-100 (Sigma T8787, France) at a

6 concentration of $0.1 \%(\mathrm{v} / \mathrm{v})$ in PBS for 10 minutes at room temperature, before incubation with

7 1:40 dilution in PBS of a solution of Alexafluor 546-labelled phalloidin (Molecular Probes

8 A22283, France) for 40 minutes at room temperature. The constructs were counterstained with

9 DAPI (Invitrogen D1306, France) at a concentration of $300 \mathrm{nM}$ in PBS for 10 minutes at room

10 temperature and investigated by confocal imaging.

Immunofluorescent labelling

13 After 3 days of culture, constructs were fixed and permeabilized according to the aforementioned

14 procedure. For saturation of non-specific binding sites, samples were incubated in a saturation

15 buffer composed of 1\% (w/v) bovine serum albumin (BSA, Sigma A3311, France) and 0.05\%

16 (v/v) Tween-20 (Sigma P1379, France) in PBS for 1 hour at room temperature. Specific primary

17 antibodies dilution (Supplementary information 2) were applied in saturation buffer overnight at

$184^{\circ} \mathrm{C}$. Then, secondary antibodies (Supplementary information 2) were diluted and applied for 1

19 hour at room temperature. The constructs were counterstained with DAPI at a concentration of

$20300 \mathrm{nM}$ in PBS for 10 minutes at room temperature and investigated by confocal imaging. For

21 negative controls, primary antibodies were excluded. Images were taken at the Centre

22 Technologique des microstructures (University of Lyon, France) on a Zeiss LSM800 confocal 23 microscope. 


\section{Transmission Electron Microscopy (TEM) Imaging}

2 Bioprinted constructs were washed twice with phosphate buffer (PB, 0.1M) and subsequently

3 incubated with 2.5\% (v/v) glutaraldehyde (Sigma G5882, France) in PB for 2 hours at room

4 temperature and finally washed again 3 times with PB solution. After dehydration, the samples

5 were infiltrated, embedded in epoxy resin and polymerized for 3 days at $56^{\circ} \mathrm{C}$. Ultrathin sections

6 (70-80 nm) were collected on Formvar-coated slot copper grids (Electron Microscopy Sciences),

7 counterstained with 7\% uranyl acetate in methanol solution and then in Reynolds' lead citrate.

8 Images were taken at the Centre Technologique des Microstructures (University of Lyon, France)

9 on a TEM Philips CM120 at $80 \mathrm{kV}$ using a CCD camera GATAN Orius 200.

\section{hVEGF ELISA quantification}

12 hVEGF was quantified in culture supernatants of bioprinted constructs after 3 days of culture using

13 a specific hVEGF ELISA Kit (Invitrogen KHG0111, France), according to manufacturer's

14 protocol. Briefly, $100 \mu \mathrm{l}$ of each supernatant were deposited on anti-human VEGF coated 96-well

15 plates and stand for binding for 2 hours at room temperature. Unbound biological components

16 were washed out using manufacturer's washing buffer. $100 \mu$ of anti-hVEGF biotin-conjugated

17 solution were then deposited into each well and incubated for 1 hour at room temperature before

18 being washed 4 times with washing buffer to remove unbound biotin-conjugated anti-hVEGF

19 antibody. $100 \mu \mathrm{l}$ of streptavidin-HRP were then added to each well and incubated for 30 minutes

20 at room temperature. During this step, streptavidin-HRP binds to the anti-hVEGF biotin-

21 conjugated antibody. Finally, $100 \mu \mathrm{l}$ of stabilized chromogen were added to each well and

22 incubated for 30 minutes at room temperature. The enzymatic reaction was terminated by the 
1 addition of $100 \mu \mathrm{L}$ of manufacturer's stop solution and the absorbance of each well measured at $2450 \mathrm{~nm}$.

$4 \quad$ RESULTS

6 To simulate Bruch's Membrane, a 5-20 $\mu \mathrm{m}$ thick GelMa layer was coated on the surface of circular

7 glass coverslips before seeding with RPE cells. Since we applied GelMa with high degree of

8 substitution (80\%), we did not experience the toxicity problem caused by unreacted methacrylic

9 anhydride and oligo methacrylic acid byproducts (Yue et al., 2015; Rose et al., 2014).

10 Figure 1-A compares viability of RPE cells cultured on GelMa layer and on classical tissue

11 culture plates (TCPs). As can be seen, RPE cells were viable and proliferate on GelMa layer over

12 the studied one-week culture time.

13 Moreover, cell population significantly increased when culture was performed on GelMa coating

$14(\mathrm{p} \leq 0.05)$, proof of the positive impact of generating a Brunch-like membrane from GelMa for RPE

15 layer sheet development. As a next step, RPE cells were labeled with PKH67 in order to directly

16 monitor cells after inkjet bioprinting. The effect of this long lasting cell staining upon growth was

17 first evaluated. Figure 1-B presents the growth comparison results between labelled PKH67 and

18 non-labelled RPE cells. As a matter of fact, a negative effect of the labelling was observed in the

19 early stages (day 1) of the RPE sheet development which was completely recovered after 3 days

20 of culture $(\mathrm{p} \leq 0.05)$

21 In second set of experiments, inkjet bioprinting effect upon the RPE sheet development on GelMa

22 membrane was evaluated. To do so, freshly dissociated RPE were bioprinted on Brunch-like 23 membrane and the behavior and growth of the cell population monitored. Figure 1-C depicts the 
1 actual $80 \mu \mathrm{m}$ nozzle very end filled with suspended RPE during inkjet bioprinting. The size of the

2 suspended cells $(15-20 \mu \mathrm{m})$ appears compatible with the nozzle size but with a clear limitation in

3 term of number of cells being able to flow concomitantly through the nozzle aperture without

4 clogging or strong loss of viability due to the presence of high shear stress.

5 Cell growth was then evaluated on both bioprinted and classically seeded RPE $\left(5.10^{4}\right.$ cells per

$6 \mathrm{~cm}^{2}$ ). As can be seen in Figure 1-D, a significant discrepancy in the metabolic activity rate of the

7 bioprinted RPE was observed when compared to classically seeded cells. Thus, after 7 days of

8 culture, the bioprinted RPE sheet which had shown a faster/development in the 1-3 days period,

9 evidenced a $25 \%$ lower metabolic activity.

10 Finally, Figures 1-E and -F present the surface of bioprinted RPÉ on GelMa Bruch-like membrane

11 after 7 days on culture. Cells were distributed homogeneously within the printed area.

12 Microphthalmia-associated transcription factor (MITF) and tight junction-associated protein

13 (ZO1) labelling were performed in order to evaluate the functional maturation of the bioprinted

14 RPE sheets. As can be seen, ZO1 was observed all over the obtained cell sheet, proof of the

15 maturation of the sheet into a dense epithelial monolayer (Li and Poznansky, 1990). Moreover, the

16 presence of MITF marker was also evidenced all over the sheet, proof of a fully functional and

17 stable RPE layer. 

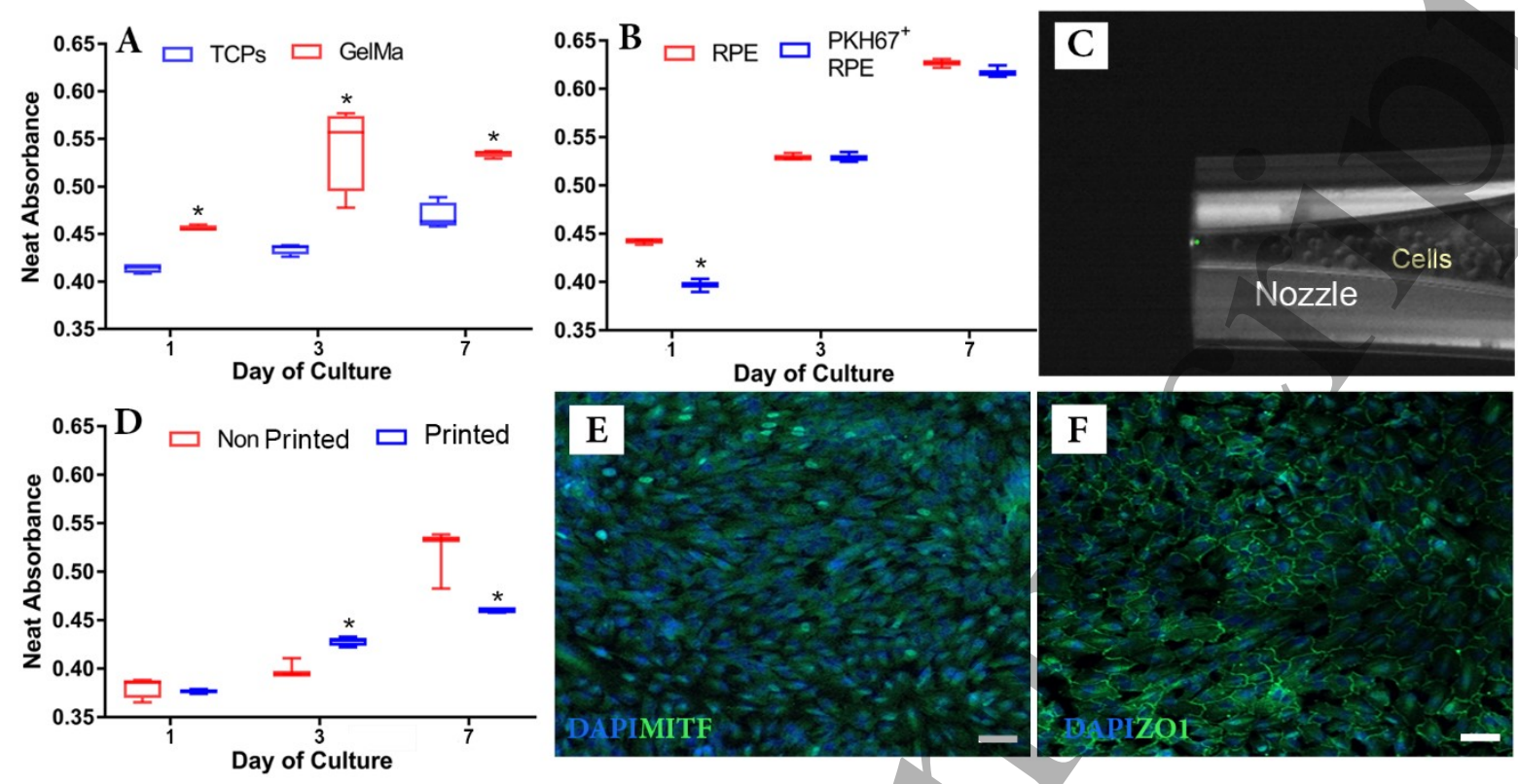

\section{F}

Figure 1. A) RPE population growth on GelMa coated layer and Tissue Culture plates (TCPs). B)

3 RPE population growth of PKH67 labeled and non-labeled RPE cells. C) Close-up image of the

4 bioprinted nozzle filled with RPE cells. D) Growth of bioprinted and seeded RPE cells on GelMa.

5 E) MITF labelling (green, counter staining with DAPI in blue) showing the presence of

Microphthalmia-associated Transcription Factor all over the RPE bioprinted sheet on GelMa

7 brunch-like membrane. F) ZO1 labelling (green, counter staining with DAPI in blue) showing the

8 distribution of tight junctions all over the RPE bioprinted sheet on GelMa brunch-like membrane.

9 Asterisks represent significant difference between groups in each day at $p \leq 0.05$. Scale bars: 50

$10 \mu m(E \& F)$.

\section{Isolation of enriched rod and cone photoreceptors}

13 In order to be able to produce full retina tissues through inkjet bioprinting, photoreceptors shall be

14 deposited at the surface of the bioprinted RPE sheet. These photoreceptor cells (PRs) were isolated

15 from pig eyes according to the protocol depicted in Figure 2-A. Following isolation, enriched 
1 population of rod and cone photoreceptors were observed by phase contrast imaging and identified

2 through immunochemical labelling using retinal cell-type-specific antibodies (Figure 2-B). Phase

3 contrast images clearly indicate a mixed population of dense cells with various shapes such as

4 round or elongated forms, typical of rod and cone retinal cells. Nevertheless, it was clear from

5 these images that the obtained isolated cells population was richer in rods than in cones.

6 Rhodopsin-based labelling of rod cells (Figure 2-C) and opsin-based labelling of cone cells

7 (Figure 2-D and 2-E) also confirmed this observation. A purity of $95 \%$ was estimated for

8 photoreceptors in the isolated population (Traverso et al., 2003).

9 Another observation was that the obtained cell suspension was composed of single isolated cells,

10 without any identified multicellular large size aggregates which might have been incompatible

11 with the $80 \mu \mathrm{m}$ diameter inkjet bioprinting nozzle.

A

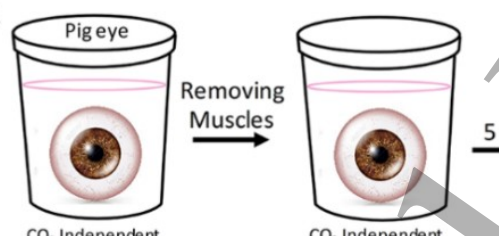

$\mathrm{CO}_{2}$ Independent

$\mathrm{CO}_{2}$ Independent
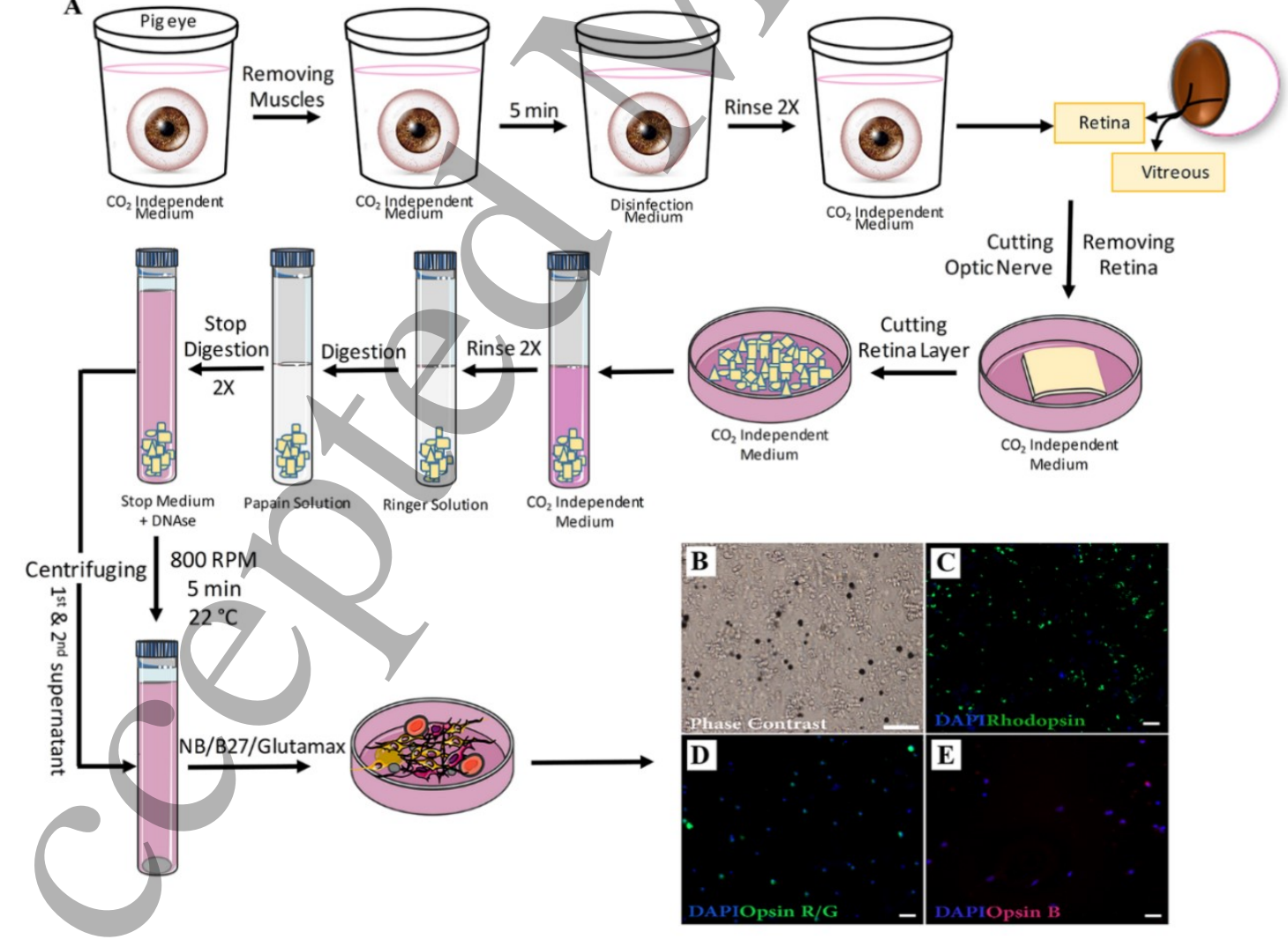
1 Figure 2. A) Schematic of photoreceptors isolation steps from pig eye. B) Phase contrast image

2 of isolated photoreceptors. C) Fluorescence image of isolated photoreceptors labelled with anti-

3 rhodopsin antibodies. C) Fluorescence image of isolated photoreceptors labelled with anti-opsin

4 R/G antibodies. E) Fluorescence image of isolated photoreceptors labelled with anti-opsin B

5 antibodies. Nuclei were counterstained with DAPI (blue). Scale bars: $50 \mu m(B)$ and $20 \mu m(C$ $6 \quad E)$

Mimicking Bruch's membrane/RPE/PRs complex through layer-by-layer cellular inkjet

10 Bioprinted RPE cell sheets on GelMa Brunch-like membranes were then used to recapitulate

11 photoreceptors/RPE complexes. To do so, freshly isolated photoreceptors were bioprinted onto 7-

12 days matured RPE cell sheets. Figure 3-A depicts the distribution of the PRs on RPE cell sheet

13 right after bioprinting. As can be seen, PRs were homogeneously distributed over the RPE cell

14 sheet, proof of the capability of the inkjet bioprinting technique to spread mature and differentiated

15 photoreceptors over a large surface (here up to $1 \mathrm{~cm}^{2}$ ). This is where the use of bioprinting has the

16 major impact: recapitulating $\mathrm{PR}$ sheet from rare, mature and freshly isolate cell. For sake of

17 comparison, PRs were also bioprinted on bare TCP surface. In that case, the bioprinted drops did

18 not spread on the surface of the underneath RPE layer, leading to non-homogeneous distribution

19 of the photoreceptors (data not show). 


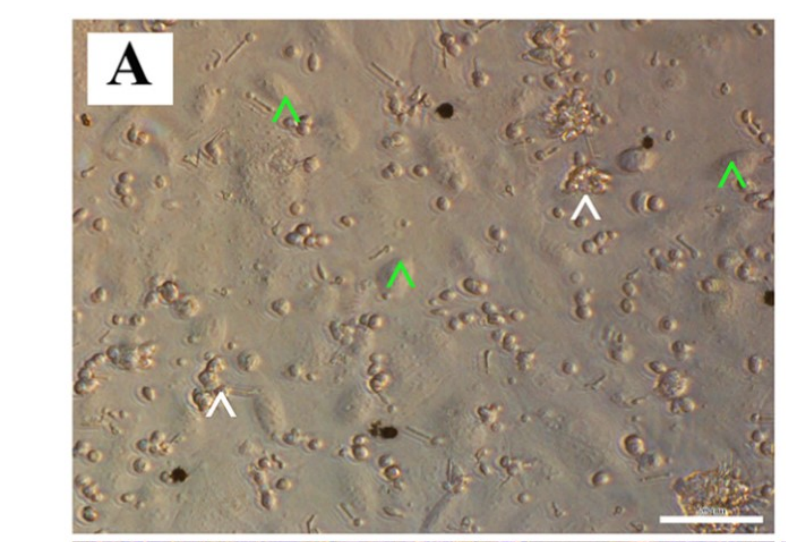

\section{B}
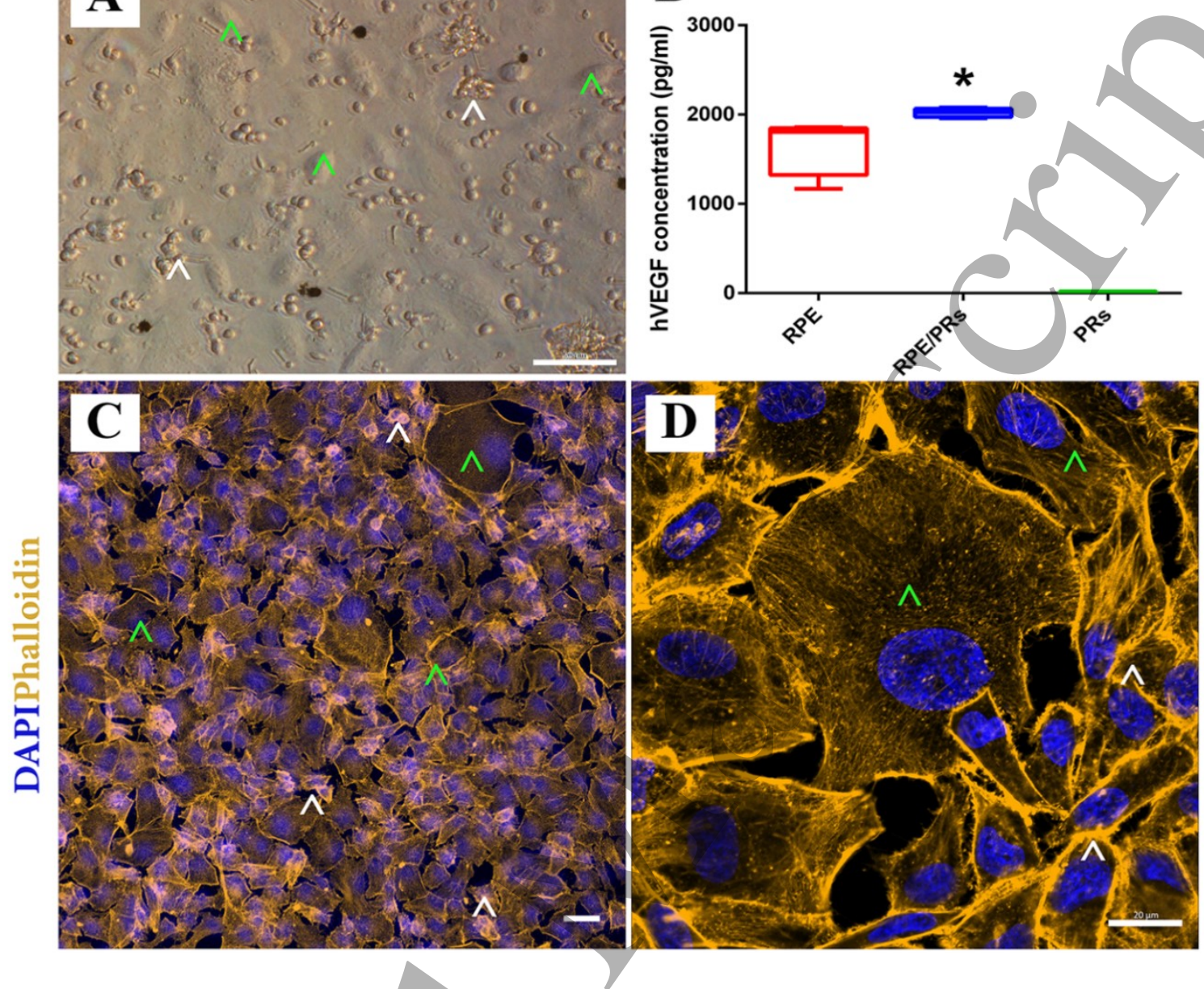

2 Figure 3. A) Phase contrast image of bioprinted PRs on RPE layer. B) Quantitative detection of

3 released hVEGF from constructs, 3 days after bioprinting. C and D) Fluorescence microscopy

4 images of cellular actin filaments (yellow) on GelMa/RPE/PRs constructs. Nuclei are

5 counterstained with DAPI (blue). Green and white arrows show RPE and PR cells, respectively.

6 Asterisk represents significant difference at $p \leq 0.05$. Scale bars: $50 \mu m(A \& C)$ and $20 \mu m(D)$.

8 hVEGF was originally recognized as an endothelial angiogenic and vasopermeability factor

9 secreted in retina by RPE cells. In order to validate that the RPE layer and the RPE/PR constructs

10 we had produced through bioprinting were still producing this important factor, hVEGF was 
1 quantified in the culture supernatants. Figure 3-B presents the results of this quantification in

2 which ng/mL levels of hVEGF were found in the RPE and RPE/PR constructs. As a control,

3 hVEGF production from PR was shown to be negligible.

4 Then, the distribution and the organization of the actin filaments within the RPE/PR/constructs

5 were evaluated through phalloidin staining and laser confocal microscopy, 3 days after PR

6 bioprinting. As can be seen in Figure 3-C and 3-D, a dense network of actin filaments was

7 evidenced with a clear identification of large and spread RPE cells (green asterisk) together with

8 small and compact PR cells (white arrows). Interaction and topology between the two cell layers,

9 i.e. RPE and PR, were not reachable using the present technique and a complementary study using

10 confocal microscopy and cell phenotype specific labelling was ímplemented.

11 ZO1, MITF, Rhodopsin, Opsin red/green, Opsin blue and PNA were then used as specific markers

12 for immunohistological analysis. Figure 4 depicts the confocal microscopy images obtained. A

13 first observation can be made about the organization of the RPE/PR constructs which appear to be

14 orientated in separated layers. Indeed, as can be seen in Figure 4-A, a clear layered organization

15 was found between the ZO1 labelled tight junction of the RPE layer and part of the DAPI labelled

16 nuclei. This observation proves then that the ZO1 labelled cells (and their nuclei) were localized

17 in the lowest part of the construct, while unlabeled cells (PRs) can be found on the upper layer of

18 the construct. The same conclusion was obtained from MITF labelled constructs (Figure 4-B).

19 Then, to ensure that PRs bioprinted on top of RPE sheet retain their initial differentiation markers,

20 3D bioprinted constructs were stained with different PR specific markers: Rhodopsin, Opsin blue,

21 Opsin red/green, and PNA. As can be seen, all these markers were identified in the obtained

22 construct (Figure 4-C, -D, -E and -F). Interestingly, these markers were found in the lower part

23 of the construct, suggesting that PR tails were partially inserted within the RPE layer. 

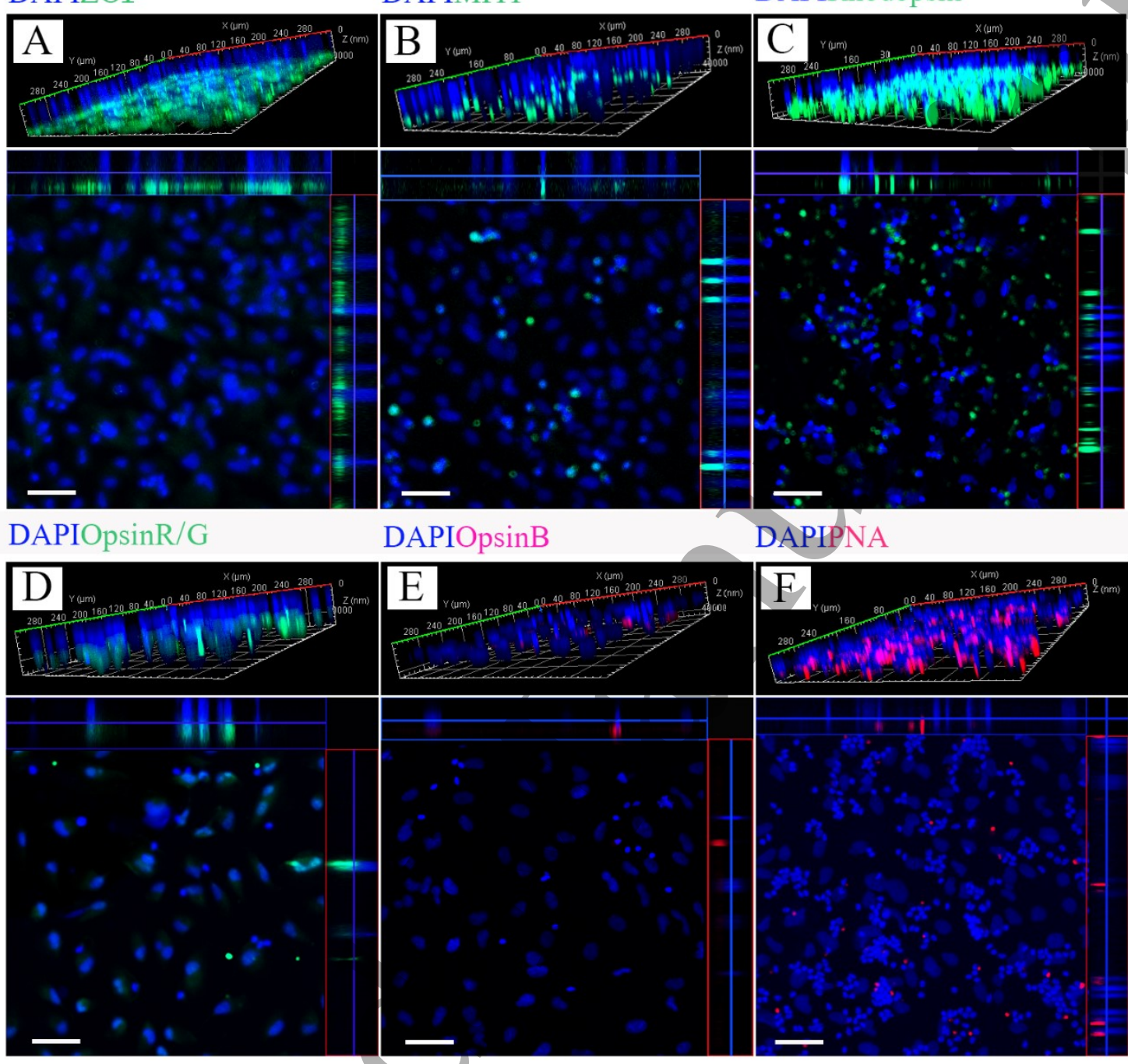

2 Figure 4. Confocal fluorescence microscopy images of bioprinted constructs. A: ZO1

3 immunolabelling. B: MITF immunolabelling. C: Rhodopsin immunolabelling. D: Opsin R/G

4 immunolabelling. E: Ospin B immunolabelling. F: PNA immunolabelling. Nuclei are

5 counterstained with DAPI (blue). The top images show 3D view and bottom images show ortho

6 view of the constructs. Scale bars: $50 \mu m(A-F)$. 

Electron Microscopy and Photoreceptor Outer Segment (POS) Phagocytosis by RPE cells after bioprinting

3 Electron microscopy of RPE cells cultured on GelMa coated substrates revealed a polarized RPE

4 sheet with many features of RPE morphology, including numerous dense bodies in the cytoplasm,

5 and apical electron-dense structures (Figure 5-A). Basolateral infolds (Bi) in RPE cells adjacent

6 to the GelMa membrane and adherent Junctions (Aj) in apical borders of RPE cells were also

7 clearly visible (Figure 5-C). Not bioprinted control PRs, depicted in Figure 5-B, also showed the

8 expected shapes of outer segments with high electron density.

9 We assessed further the RPE-PRs interactions upon bioprinting at ultrastructural level. After 24h

10 cultivation of complete bioprinted GelMa/RPE/PRs constructs, internal structure of the 11 phagocytized POS can be observed (Figures 5-D to -F). Numerous microvilli (Mv) were also 12 observed on the apical RPE of the bioprinted final construct (Figure 5-F). 


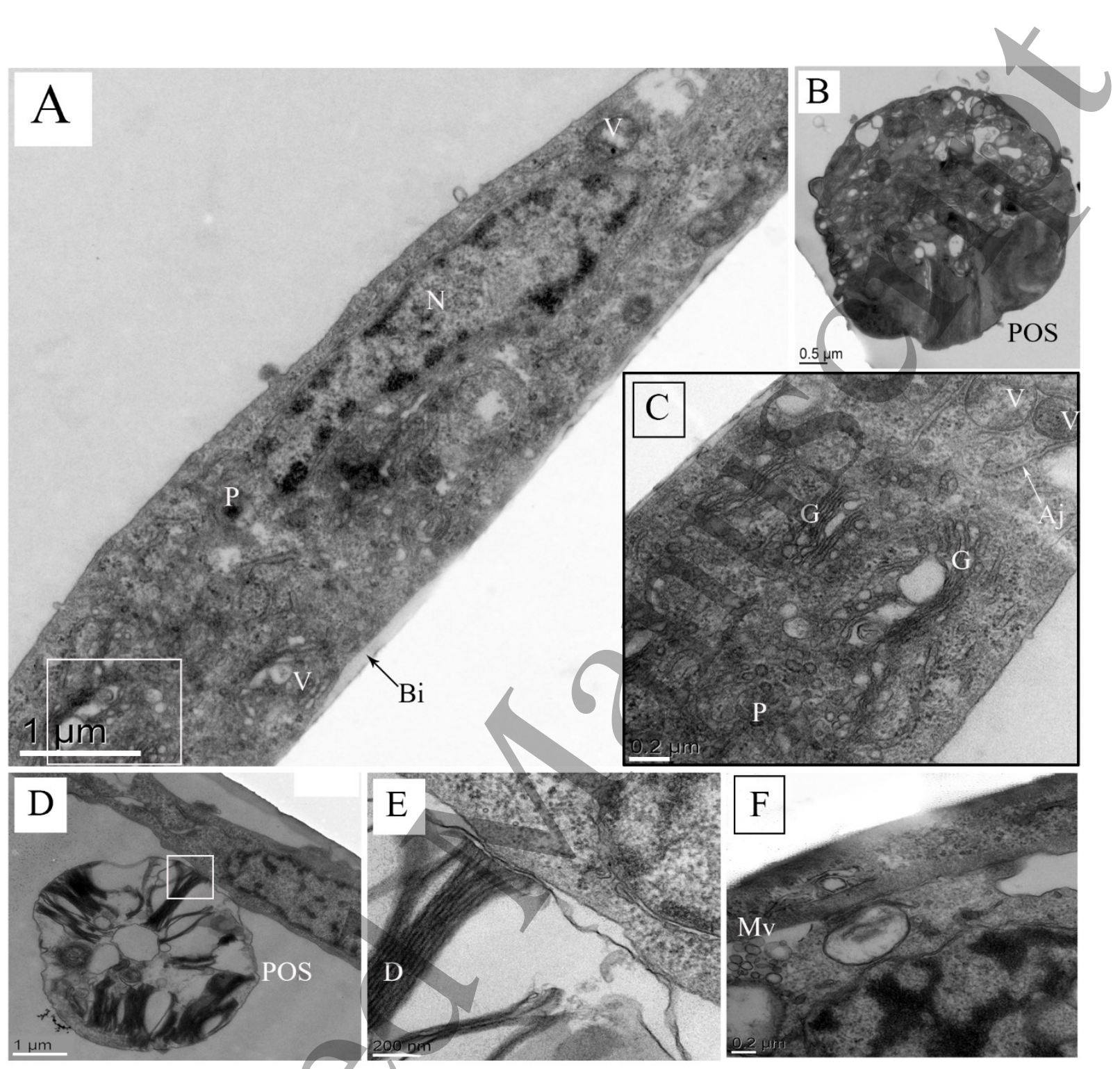

2 Figure 5. Electron micrographs depicting ultrastructures of 3D bioprinted constructs. A \& C) RPE

3 sheet, B) photoreceptor outer segment (POS) of isolated PRs, D-F) POS phagocytosis by RPE

4 sheet $24 \mathrm{~h}$ after cultivation of complete bioprinted construct.

5 V: vesicular compartments; N: nuclei; P: pigment molecules; Bi: basolateral infolds; G: Golgi; Aj:

6 adherent junctions; D: photoreceptor disks; Mv: microvilli. Scale bars: $1 \mu m(A \& D), 0.5 \mu m(B)$,

$7 \quad 0.2 \mu m(C, E \& F)$.

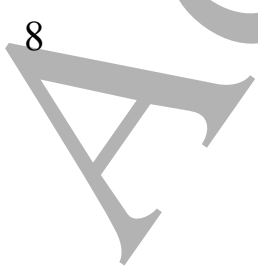




\section{DISCUSSION}

2 The retina is a complex neuro-sensory tissue responsible for primary visual signal processing. In

3 some ocular pathologies such as age-related macular degeneration and retinitis pigmentosa,

4 degeneration or atrophy of RPE cells in retina layer, directly followed by a loss of the associated

5 photoreceptor cells, lead to vision deterioration. Most of native RPE functions such as gene

6 expression profile, ability of cells to form tight junctions, polarized cytokine secretion, and ability

7 to phagocytose POS are related to 3D structure of retina (Miyagishima et al., 2017; Hsiung et al.,

8 2015). This is the main reason why multiple ongoing efforts for regeneration of damaged retina

9 have been focused on preservation of 3D layered structure of retinal cells and their secreted 10 matrix.

11 In the present study, we applied 3D inkjet bioprinting technique as a novel carrier-free method to

12 simulate retina tissue with 3D multi-layered structure, without any carrier material. In a first step,

13 a Bruch's membrane model was designed from GelMa thin layer, in order to achieve a biomimetic

14 physiological microenvironment of the native retina. This part is of upmost importance since this

15 is where the RPE layer is separated by a/membrane from the underlying fenestrated choroidal

16 capillaries of the eye. As GelMa is a gelatin derivative material, it contains significant amounts of

17 matrix metalloproteinase peptide motifs, which positively affect cellular function (Yue et al.,

18 2015). Therefore, this design was found to suit well the development of RPE cells, as observed in

19 our results (Figure 1-A). After mimicking Bruch's membrane, a piezoelectric inkjet printer was

20 used to develop a retina cell bioprinting process taking advantage of the high resolution of the

21 machine $\mathrm{X} / \mathrm{Y}$ axis $(5 \mu \mathrm{m})$ and the extremely low deposition volume (down to $180 \mathrm{pL}$ ).

22 In our carrier-free bioprinting approach, cell suspension was directly deposited during bioprinting

23 process and probably due to the absence of any other biomaterial, no clogging occurred within the 
1 capillary nozzle (Figure 1-C). Compared to extrusion method, inkjet bioprinting commonly

2 requires low viscosities to avoid nozzle clogging and control potential for jamming. Low viscosity

3 bioinks is also desirable to control fluid shear stress, known as a crucial factor that unfavorably

4 affects viability, signaling and protein expression after bioprinting (Chimene et al., 2016; Ozbolat,

5 2015). One possible reason is that, excessive stress probably dispatches cells by disrupting cellular

6 membrane (Blaeser et al., 2016).

7 During bioprinting with hydrogel cell laden bioinks, when high viscosity materials travel through

8 the extrusion nozzle, cells are expose to high levels of shear stress which affect their immediate

9 viability and ultimately their functionalities. Our hypothesis is that, short-time $(100 \mu \mathrm{s})$ exposure

10 to low levels of shear stress (Calculated using the nozzle geometry and a viscosity of $1.00 \mathrm{E}^{-03}$

11 Pa.s: Wall Shear rate: $1.31 \mathrm{E}^{+08} \mathrm{~s}^{-1}$; Wall Shear stress: 1.31E $\mathrm{E}^{+05} \mathrm{~Pa}$ (FlowTips ${ }^{\circledR}$ Program, 3d.FAB,

12 France) in our carrier-free system does not affect cell viability immediately after printing (day 1),

13 but might still induce long-term alterations in the proliferation potential of cells that survived the

14 printing process (day 7) (Figure 1-D). However, although bioprinting process has reduced RPE

15 proliferation rate over a week, the bioprinted cells adapted with process condition, survived and

16 continued their proliferation after printing.

17 Without ink materials, we were also capable of bioprinting cell densities closer to the figures of a

18 native tissue (Ozbolat, 2015). Indeed, during inkjet bioprinting, single drop ejected from the

19 nozzle has, under our experimental conditions, a volume of 300 pL. 52 drops were dispensed per

20 deposition location leading to an estimated cell density of around $93 \pm 15$ RPE cells per deposition.

21 This will be of high importance when the technique will be applied to the deposition of highly

22 differentiated non-proliferative cells such as photoreceptors. 
1 Furthermore, our approach as well helps RPE cells to stack together for formation of monolayer

2 sheet. Indeed, carrier-free deposition may also facilitate cellular maturation after bioprinting and

3 GelMa coating probably guides cells to form monolayer sheet faster. In RPE sheets, extracellular

4 matrix and secreted adhesion molecules are crucial regulators of cell behavior, which prevent cell

5 apoptosis and allow an easy manipulation during subsequent transplantation. In fact, intracellular

6 tight junction formation in RPE monolayer plays an important role incarrying out retina functions

7 (Kamao et al., 2014). Accordingly, we demonstrated that, bioprinted RPE cells positively express

8 typical membrane-associated proteins such as ZO1 and MITF, similar to that of native RPE sheet

9 in vivo (Figures 1-E and -F). These factors were identified in 3D bioprinted constructs, after Z-

10 stack 3D confocal imaging (Figures 4-A \& -B). ZO1 is a common adaptor protein associated with

11 tight junctions, anchoring junctional macromolecular complexes to cytoplasmic actin (Obert et

12 al., 2017; Liao et al., 2010). MITF is also considered as essential for terminal pigment

13 differentiation in the RPE (Westenskow et al., 2009). These proteins are polarized and actively

14 interact with other membrane proteins/receptors, cell adhesion molecules, and the cytoskeleton to

15 regulate epithelial cell morphology and assemble signaling cascades.

16 In the next step, we had isolated PRs using a well-defined protocol with the purpose of applying

17 them as the second cellular layer of bioprinted retina (Figure 2-A). PRs (rods and cones) are highly

18 specialized neurons with stacks of photosensitive disks that have special morphology and express

19 different rod- or cone-specific markers. Although, we have no direct evidence on proportion of

20 isolated rod cells to cones, based on Traverso 's protocol, isolated cells probably have rod-cone

21 ratio between 1.3 and 2 (Traverso et al., 2003). In a classic approach, we then characterized

22 isolated cells through imaging, morphological analysis and immunofluorescent labeling of light-

23 sensitive receptor proteins such as rhodopsin, opsin R/G and opsin B (Figures 2-B to $-\mathbf{E}$ ). 
1 Afterward, primary isolated PRs were bioprinted on RPE/GelMa layer to form 3D retina constructs. For RPE cells, we designed printing pattern based on our study plan. Since we needed

3 a RPE monolayer sheet, and for cells maturation we should incubate them for at least a week, we

4 decided to make a gap between RPE depositions, so that the cells had enough space for growth

5 and maturation (Supplementary Information 1). Over the studied one-week culture time, we had

6 virtually no non-RPE regions. We next showed full epithelium structure of cells by

7 immunostaining images of ZO1 marker as well as hVEGF measurement.

8 In contrast, for obtaining a PR surface coverage close to the native one, and since photoreceptor

9 cell body size is less than that of RPE cell, we had increased and optimized the number of

10 deposition location through a trial and error bioprinting of PRs on RPE/GelMa layer.

11 Once PR bioprinting feasibility was proven, we confirmed the presence of positioned PRs, 3 days

12 after bioprinting with phase-contrast imaging and cytoskeleton staining (Figures 3-A, -C and -

13 D). Although without specific staining it is difficult to distinguish PRs from underlying RPE cells,

14 expected morphology of PRs with long outer segments are evident in Figure 3-A.

15 As another part of our study, we investigated the possibility that human RPE cells secrete 16 angiogenic factors such as hVEGF after bioprinting process (Figure 3-B). hVEGF protein is a 17 potent endothelial factor which promotes angiogenesis and its optimal levels is essential for 18 maintaining choriocapillaris and choroidal vessels in vivo (Adamis et al., 1993).

19 Obviously, hVEGF must be secreted within a defined concentration range to be biologically

20 functional. Blaauwgeers et al. have reported that primary RPE cells secrete between $0.2 \mathrm{ng} / \mathrm{ml}$ on 21 their apical side to $11 \mathrm{ng} / \mathrm{ml}$ on their basal side (Blaauwgeers et al., 1999). There numbers

22 correlated well with our results since the concentration of hVEGF found in the construct culture 23 medium in our experimental conditions was $2 \pm 0.06 \mathrm{ng} / \mathrm{ml}$. 
1 ARPE19 cells retained their growth-adapted phenotype when cultured under standard conditions, 2 indicated by expended cell morphology and interdigitated cell-cell junctions. The exact 3 constituents of the proteins released from RPE cells are available in the literatures. For example,

4 McLenachan et al. reported that, decellularized ARPE19-ECM displayed immunoreactivity for

5 proteins found in the inner layers of Bruch's membrane, including fibronectin, vitronectin,

6 collagens IV, and V as well as laminin- $\alpha 5$ (McLenachan et al., 2017). Here, we hypothesized that

7 this effective production of secreted hVEGF from bioprinted RPE cells may be due to known roles

8 of 3D structure of ECM. Indeed, with bioprinting, we had organized RPE cells in a layered way

9 on top of a GeMa Bruch's like membrane which can provide mechanical support for next

10 angiogenesis. In other words, GelMa can, in vitro, induce recapitulation of the native RPE

11 microenvironment and provided enriched ECM conditions for RPE cell culture.

12 Such a role for 3D matrix has been previously confirmed by others (Mousa et al., 1999; Farjood

13 and Vargis, 2018) and is based on the observation that ECM of RPE cells can cause increased

14 secretion of angiogenic growth factors. Despite, certain importance of hVEGF for stimulating

15 vascular permeability, RPE-secreted hVEGF has also neuroprotective effects for neural retina and

16 are anti-apoptotic agent for retinál neurons (Nishijima et al., 2007; Kilic et al., 2006).

17 A step forward in the present study has been the immunofluorescent detection of specific

18 molecular markers within our retina constructs. Thus, we were able to detect considerable

19 expression levels of ZO1 and MITF as membrane-associated proteins of RPE layer but also

20 rhodopsin, opsin $\mathrm{R} / \mathrm{G}$ and opsin B as light-sensitive proteins expressed in rod and cone outer

21 segments, and PNA as cone ECM marker after bioprinting (Figure 4). One of the main results

22 here is the fact that some nuclei are co-localized with RPE markers and some co-localized with

23 PR markers. Also, some nuclei are in the lower part of the construct and other in the upper part. 
1 Therefore, part of immunostaining results are difficult to analyze. However, these data indicate

2 that bioprinting process reliably and robustly produces a GelMa/RPE/PRs complex with adequate

3 functionality comparable with that of native retina. As cultivation and maintenance of primary

4 isolated PRs for long-term periods is a key challenge for in vitro studies, we just experimented

5 above behaviors 3 days after incubation. It was well known that, after dissociation from retinal

6 environment, isolated PRs lose their functional integrity in the primary cell culture (Reidel et al.,

7 2006; Fintz et al., 2003).

8 Different studies on retinal cells recommend that retinal PRs require specific trophic factors or

9 feeder layers for long-term culture (Fintz et al., 2003). Interactions between PRs and neighboring

10 Müller glial cells is also of important for PRs function and survival (Vecino et al., 2016).

11 Therefore, long-term incubation of bioprinted constructs in the native environment of PRs should

12 be done in future to investigate potential value of bioprinting for studying sight-threatening

13 diseases.

14 The present observation of the immunofluorescent labelled constructs through confocal

15 microscopy is one of the first attempt in assessing biological characterization of bioprinted retina

16 construct. Indeed, even if in one of the few available articles about retina bioprinting, Shi et al.

17 reported excellent viability of both ARPE-19 and Y79 cell lines after bioprinting (Shi et al., 2017),

18 no biological assessment related to the specific functionality of bioprinted cells was performed.

19 Here, we have shown for the first time that both bioprinted RPE and PRs expressed essential

20 transcription factors. This observation is crucial for the validation of functional GelMa/RPE/PRs

21 bioprinted constructs for future clinical applications.

22 To further expand our findings about functionality of bioprinted constructs, we assessed cells'

23 microstructure through electron microscopy (Figure 5) and showed specific phagocytosis of POS 
1 by apical surface of RPE cells. Just like in the case of the biological characterization of bioprinted

2 retinal cells, previous studies have not addressed the phagocytosis of POS after bioprinting. RPE

3 cells phagocyte POS fragments to remove the photo-oxidative residual bodies that accumulate

4 daily during phototransduction process (Penberthy et al., 2018). Many studies have confirmed that any dysfunction in POS phagocytosis pathway may lead to different retinal pathologies and

6 eventual blindness (Carr et al., 2009; Nandrot et al., 2004; Mao and Finnemann, 2012; Penberthy

7 et al., 2018). For example, deficiency or mutation of phagocytosis associated molecular factors

8 such as MerTK and PtdSer receptors may cause rapid RPE degradation and retinitis pigmentosa

9 pathology (Burstyn-Cohen et al., 2012; Ostergaard et al,,2011; Penberthy et al., 2018). Expected

10 morphology of RPE cells in TEM images and development of epithelial monolayer, which

11 previously was established by ZO1 and MITF protein expressions, are of great importance in

12 phagocytic capacity of RPE cells after bioprinting. Thís topic has been mentioned in literatures,

13 when relationship between POS phagocytosis pathway and RPE differentiation has been studied

14 (Mao and Finnemann, 2012; Mazzoni et al., 2014). With further evaluation of the ultrastructure

15 images of 3D bioprinted constructs, adjacency of POS disk fragments with apical microvilli of

16 RPE layer were observed (Figures 5-D to -F). Presence of RPE microvilli is essential for

17 functional in vivo phagocytosis and is a main challenge during in vitro simulation of this

18 phenomenon (Feng et al., 2002). Here we can claim that, using bioprinting approach, we succeeded

19 in binding PRs' outer segment to RPE apical microvilli, recapitulating finely an in vivo

20 phenomenon where direct contact of outer segments with RPE microvilli contributes to

21 phagocytosis.

22 In summary, we successfully engineered part of the complex 3D structure of the retina using inkjet 23 bioprinting technology in a carrier-free approach. Layer-by-layer printing of photoreceptor cells 
1 on RPE monolayer did not alter cells' biological functions such as morphology, viability and

2 expression of specific proteins, leading to an engineered construct with considerable resemblance

3 to native retina. While no direct tests were conducted to assay outer segment phagocytosis and

4 visual cycle activity, the protein expression data and microstructure imaging suggested that after

5 bioprinting, both RPE and PRs were capable of performing specific retinal functions. These

6 functional studies of the obtained constructs will be have to be examined in depth in the future. 7

8 AUTHOR INFORMATION

9 Corresponding Authors

10 *E-mails: Elahe Masaeli (elahe.masaeli@royaninstitute.org) or Christophe Marquette

11 (christophe.marquette@univ-lyon1.fr)

12

\section{Author Contributions}

14 Conceived and designed the experiments: EM VF FK MHN-E CM. Performed the experiments:

15 EM CM. Analyzed the data: EM CM FK. Wrote the paper: EM CM SP.

\section{ACKNOWLEDGMENT}

18 Confocal and Transmission Electron Microscopy experiments were performed at the Centre

19 Technologique des Microstructures (University of Lyon, France) under the supervision of

20 Christelle Fabrer-Boulé and Veronica La Padula.

21

22 FUNDING SOURCES 


\section{REFERENCES}

Adamis A, Shima D, Yeo K-T, Yeo T, Brown L, Berse B, Damore P and Folkman J 1993 Synthesis and secretion of vascular permeability factor/vascular endothelial growth factor by human retinal pigment epithelial cells Biochem Biophys Res Commun 193 631-8

Blaauwgeers H G, Holtkamp G M, Rutten H, Witmer A N, Koolwijk P, Partanen T A, Alitalo K, Kroon M E, Kijlstra A and van Hinsbergh V W 1999 Polarized vascular endothelial growth factor secretion by human retinal pigment epithelium and localization of vascular endothelial growth factor receptors on the inner choriocapillaris: evidence for a trophic paracrine relation The American journal of pathology 155 421-8

Blaeser A, Duarte Campos D F, Puster U, Richtering W, Stevens M M and Fischer H 2016 Controlling shear stress in 3D bioprinting is a key factor to balance printing resolution and stem cell integrity Advanced healthcare materials 5 326-33

Burstyn-Cohen T, Lew E D, Través P G, Burrola P G, Hash J C and Lemke G 2012 Genetic dissection of TAM receptor-ligand interaction in retinal pigment epithelial cell phagocytosis Neuron 76 1123-32

Carr A-J, Vugler A, Lawrence J, Chen L L, Ahmado A, Chen F K, Semo M a, Gias C, da Cruz L and Moore H D 2009 Molecular characterization and functional analysis of phagocytosis by human embryonic stem cell-derived RPE cells using a novel human retinal assay Mol Vis 15283

Chimene D, Lennox K K, Kaunas R R and Gaharwar A K 2016 Advanced bioinks for 3D printing: a materials science perspective Ann Biomed Eng 44 2090-102

da Silva G R, Junior A D C, Saliba J B, Berdugo M, Goldenberg B T, Naud M C, Ayres E, Orefice R L and Cohen F B 2011 Polyurethanes as supports for human retinal pigment epithelium cell growth Int J Artif Organs 34 198-209

De Maria C, Vozzi G and Moroni L 2017 Multimaterial, heterogeneous, and multicellular three-dimensional bioprinting Mrs Bull 42 578-84

Dias A, Kingsley D and Corr D 2014 Recent Advances in Bioprinting and Applications for Biosensing Biosensors 4111

Farjood F and Vargis E 2018 Novel devices for studying acute and chronic mechanical stress in retinal pigment epithelial cells Lab Chip 18 3413-24

Feng W, Yasumura D, Matthes M T, LaVail M M and Vollrath D 2002 Mertk triggers uptake of photoreceptor outer segments during phagocytosis by cultured retinal pigment epithelial cells J Biol Chem 277 17016-22

Fintz A-C, Audo I, Hicks D, Mohand-Said S, Léveillard T and Sahel J 2003 Partial characterization of retinaderived cone neuroprotection in two culture models of photoreceptor degeneration Investigative ophthalmology \& visual science $\mathbf{4 4}$ 818-25

Guillotin B and Guillemot F 2011 Cell patterning technologies for organotypic tissue fabrication Trends Biotechnol 29 183-90

Hsiung J, Zhu D and Hinton D R 2015 Polarized Human Embryonic Stem Cell-Derived Retinal Pigment Epithelial Cell Monolayers Have Higher Resistance to Oxidative Stress-Induced Cell Death Than Nonpolarized Cultures Stem cells translational medicine 4 10-20 
Jorge I R-D, Bimeng Z, Daniel R, Zhi-dong S and Tao X 2012 High throughput miniature drug-screening platform using bioprinting technology Biofabrication 4035001

Kamao H, Mandai M, Okamoto S, Sakai N, Suga A, Sugita S, Kiryu J and Takahashi M 2014 Characterization of human induced pluripotent stem cell-derived retinal pigment epithelium cell sheets aiming for clinical application Stem cell reports 2 205-18

Kilic Ü, Kilic E, Järve A, Guo Z, Spudich A, Bieber K, Barzena U, Bassetti C L, Marti H H and Hermann D M 2006 Human vascular endothelial growth factor protects axotomized retinal ganglion cells in vivo by activating ERK-1/2 and Akt pathways J Neurosci 26 12439-46

Kolesky D B, Truby R L, Gladman A S, Busbee T A, Homan K A and Lewis J A 2014 3D bioprinting of vascularized, heterogeneous cell-laden tissue constructs Advanced materials 26 3124-30

Li C and Poznansky M J 1990 Characterization of the ZO-1 protein in endothelial and other cell lines J Cell Sci 97 231-7

Liao J-L, Yu J, Huang K, Hu J, Diemer T, Ma Z, Dvash T, Yang X-J, Travis G H and Williams D S 2010 Molecular signature of primary retinal pigment epithelium and stem-cell-derived RPE cells Hum Mol Genet 19 4229-38

Luo X, Heidinger V r, Picaud S, Lambrou G, Dreyfus H, Sahel J and Hicks D 2001 Selective excitotoxic degeneration of adult pig retinal ganglion cells in vitro Investigative ophthalmology \& visual science 42 1096-106

Mao Y and Finnemann S C 2012 Retinal Degeneration: Springer) pp 285-95

Mazzoni F, Safa H and Finnemann S C 2014 Understanding photoreceptor outer segment phagocytosis: use and utility of RPE cells in culture Exp Eye Res 126 51-60

McLenachan S, Hao E, Zhang D, Zhang L, Edel M and Chen F 2017 Bioengineered Bruch's-like extracellular matrix promotes retinal pigment epithelial differentiation Biochemistry and biophysics reports 10 178-85

Miyagishima K J, Wan Q, Miller S S and Bharti K 2017 A basis for comparison: sensitive authentication of stem cell derived RPE using physiological responses of intact RPE monolayers Stem cell and translational investigation 4

Mokhtarinia K, Nourbakhsh M S, Masaeli E, Entezam M, Karamali F and Nasr-Esfahani M H 2018 Switchable phase transition behavior of thermoresponsive substrates for cell sheet engineering Journal of Polymer Science Part B: Polymer Physics

Mousa S A, Lorelli W and Campochiaro P A 1999 Role of hypoxia and extracellular matrix-integrin binding in the modulation of angiogenic growth factors secretion by retinal pigmented epithelial cells $J$ Cell Biochem 74 135-43

Nandrot E F, Kim Y, Brodie S E, Huang X, Sheppard D and Finnemann S C 2004 Loss of synchronized retinal phagocytosis and age-related blindness in mice lacking $\alpha v \beta 5$ integrin J Exp Med 200 1539-45

Nishijima K, Ng Y-S, Zhong L, Bradley J, Schubert W, Jo N, Akita J, Samuelsson S J, Robinson G S and Adamis A P 2007 Vascular endothelial growth factor-A is a survival factor for retinal neurons and a critical neuroprotectant during the adaptive response to ischemic injury The American journal of pathology 171 53-67

Obert E, Strauss R, Brandon C, Grek C, Ghatnekar G, Gourdie R and Rohrer B 2017 Targeting the tight junction protein, zonula occludens-1, with the connexin43 mimetic peptide, $\alpha \mathrm{CT} 1$, reduces VEGFdependent RPE pathophysiology Journal of Molecular Medicine 95 535-52

Ostergaard E, Duno M, Batbayli M, Vilhelmsen K and Rosenberg T 2011 A novel MERTK deletion is a common founder mutation in the Faroe Islands and is responsible for a high proportion of retinitis pigmentosa cases Mol Vis 171485

Ozbolat I T 2015 Scaffold-based or scaffold-free bioprinting: competing or complementing approaches? Journal of Nanotechnology in Engineering and Medicine 6024701 
Penberthy K K, Lysiak J J and Ravichandran K S 2018 Rethinking phagocytes: Clues from the retina and testes Trends Cell Biol 28 317-27

Pourchet LJ, Thepot A, Albouy M, Courtial E J, Boher A, Blum L J and Marquette C A 2017 Human Skin 3D Bioprinting Using Scaffold-Free Approach Adv Healthc Mater 61601101

Ramsden C M, Powner M B, Carr A-J F, Smart M J, da Cruz L and Coffey P J 2013 Stem cells in retinal regeneration: past, present and future Development 140 2576-85

Reidel B, Orisme W, Goldmann T, Smith W C and Wolfrum U 2006 Photoreceptor vitality in organotypic cultures of mature vertebrate retinas validated by light-dependent molecular movements Vision research 46 4464-71

Rose J, Pacelli S, Haj A, Dua H, Hopkinson A, White L and Rose F 2014 Gelatin-based materials in ocular tissue engineering Materials 7 3106-35

Shi P, Tan E Y S, Yeong W Y and Laude A 2017 Hybrid three-dimensional (3D) bioprinting of retina equivalent for ocular research

Shi P, Tan Y S E, Yeong W Y, Li H Y and Laude A 2018 A bilayer photoreceptor-retinal tissue model with gradient cell density design: A study of microvalve-based bioprinting Journal of tissue engineering and regenerative medicine 12 1297-306

Singh R, Cuzzani O, Binette F, Sternberg H, West M D and Nasonkin I O 2018 Pluripotent Stem Cells for Retinal Tissue Engineering: Current Status and Future Prospects Stem Cell Reviews and Reports 14 463-83

Tasoglu S and Demirci U 2013 Bioprinting for stem cell research Trends Biotechnol 31 10-9

Tomita M, Lavik E, Klassen H, Zahir T, Langer R and Young M $J 2005$ Biodegradable polymer composite grafts promote the survival and differentiation of retinal progenitor cells Stem Cells 23 1579-88

Traverso V r, Kinkl N, Grimm L, Sahel J and Hicks D 2003 Basic Fibroblast and Epidermal Growth Factors Stimulate Survival in Adult Porcine Photoreceptor Cell Cultures Investigative Ophthalmology \& Visual Science $\mathbf{4 4}$ 4550-8

Trese M, Regatieri C V and Young M J 2012 Advances in Retinal Tissue Engineering Materials 5108

Tropepe V, Coles B L, Chiasson B J, Horsford D J, Eliá A J, McInnes R R and van der Kooy D 2000 Retinal stem cells in the adult mammalian eye Science 287 2032-6

Umemoto T, Yamato M, Nishida K and Okano T 2013 Regenerative medicine of cornea by cell sheet engineering using temperature-responsive culture surfaces Chin Sci Bull 58 4349-56

Vecino E, Rodriguez F D, Ruzafa N, Pereiro X and Sharma S C 2016 Glia-neuron interactions in the mammalian retina Progress in retinal and eye research 51 1-40

Westenskow P, Piccolo S and Fuhrmann S 2009 -catenin controls differentiation of the retinal pigment epithelium in the mouse optic cup by regulating Mitf and Otx2 expression Development 1362505 10

Xu T, Zhao W, Zhu J M, Albanna M Z, Yoo J J and Atala A 2013 Complex heterogeneous tissue constructs containing multiple cell types prepared by inkjet printing technology Biomaterials 34 130-9

Yaji N, Yamato M, Yang J, Okano T and Hori S 2009 Transplantation of tissue-engineered retinal pigment epithelial cell sheets in a rabbit model Biomaterials 30 797-803

Yang J, Yamato M, Nishida K, Hayashida Y, Shimizu T, Kikuchi A, Tano Y and Okano T 2006 Corneal epithelial stem cell delivery using cell sheet engineering: not lost in transplantation J Drug Target 14 471-82

Yao J, Tao S L and Young M J 2011 Synthetic Polymer Scaffolds for Stem Cell Transplantation in Retinal Tissue Engineering Polymers 3899

Yu Z, Rui Y, Liliang O, Hongxu D, Ting Z, Kaitai Z, Shujun C and Wei S 2014 Three-dimensional printing of Hela cells for cervical tumor model in vitro Biofabrication 6035001 
1 Yue K, Trujillo-de Santiago G, Alvarez M M, Tamayol A, Annabi N and Khademhosseini A 2015 Synthesis,

2

3

4

5

6 properties, and biomedical applications of gelatin methacryloyl (GelMA) hydrogels Biomaterials 73 254-71

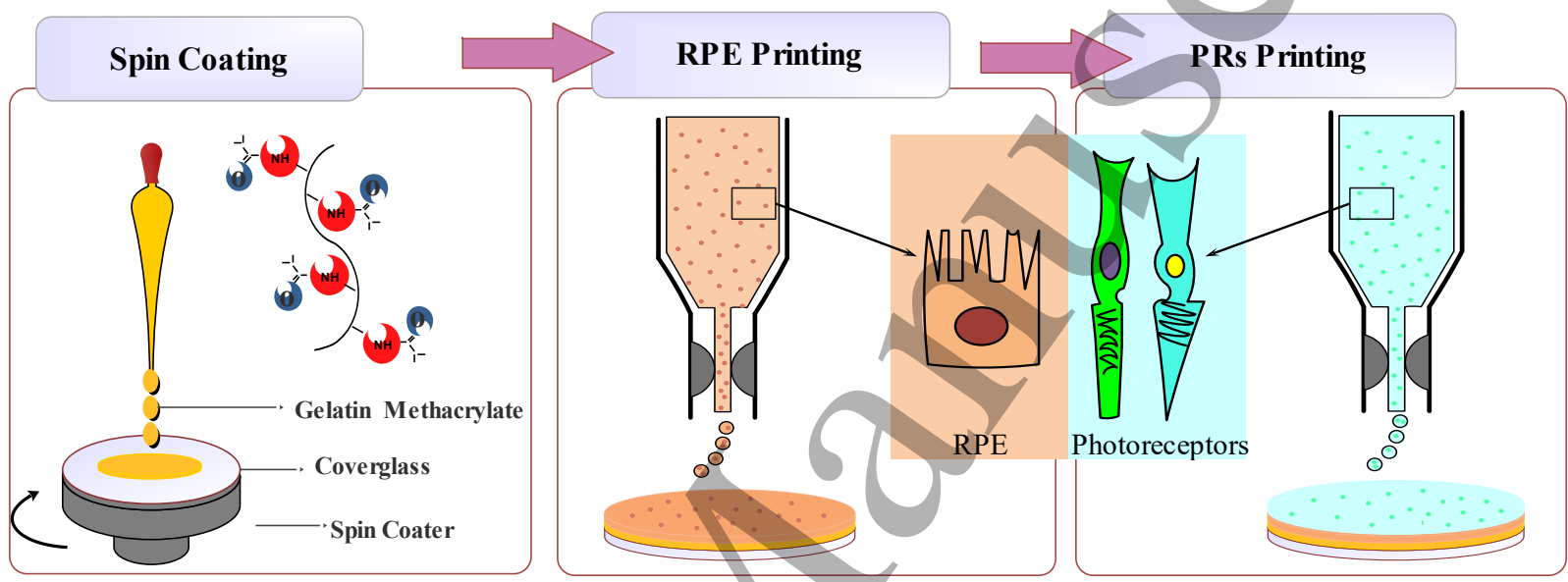

8 In this study, a unique inkjet-based bioprinter was applied to deposit matured and differentiated

9 photoreceptors (PRs) on retina pigmented epithelium (RPE) layer in a predefined arrangement to 10 create complex double-cell sheets. 